\section{Weiterbildung: Ab Juli gibt es höhere Förderzuschüsse}

umzusetzen. In der Hauptvereinbarung werden die gesetzlichen Vorgaben erfüllt, um in einem nächsten Schritt dann zu beraten, welche Einrichtungen gefördert werden sollen. Gemäß den Grundsätzen aus der Hauptvereinbarung kann die Förderung im Rahmen der Weiterbildung sofort starten. Mindestens 7.500 Weiterbildungsstellen im ambulanten und stationären Bereich sollen die Kostenträger pro Jahr fördern. Stellenbegrenzungen durch KVen sind nicht mehr zulässig.

\section{MMW-KOMMENTAR}

Der Gehaltszuschuss für einen Weiterbildungsassistenten in der Allgemeinmedizin erhöht sich ab dem 1. Juli auf 4.800 Euro brutto im Monat bei zwölf Monatsgehältern pro Jahr. Die Praxis wird darüber hinaus verpflichtet, das Gehalt des Arztes in Weiterbildung auf das Gehaltsniveau einer stationären Weiterbildungsstelle anzuheben. In unterversorgten Regionen gibt es einen weite-

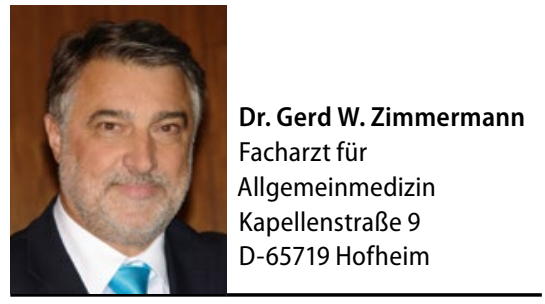

ren Gehaltszuschuss von monatlich 500 Euro, in drohend unterversorgten Regionen immerhin noch 250 Euro.

Die Mindestförderdauer für eine Vollzeitstelle beträgt drei Monate. Teilzeitstellen mit mindestens der Hälfte der regelmäßigen Arbeitszeit werden ebenfalls gefördert. Im Falle einer Verbundweiterbildung sind kürzere $A b$ schnitte möglich, sofern diese in der Weiterbildungsordnung vorgesehen sind. Auch Teilzeitstellen bis hinunter zu 12 Wochenstunden können gefördert werden, wenn sie gemäß der regionalen Weiterbildungsordnung anerkannt werden. Auch die maximale Förderdauer leitet sich aus der Weiterbildungsordnung ab, allerdings sollte die Weiterbildung planmäßig innerhalb von fünf Jahren abgeleistet werden.

Da ein Begrenzungsverbot für die Förderung der allgemeinmedizinischen Weiterbildung besteht, greift ein bundesweiter Finanzausgleich zwischen den KVen, sobald die bevölkerungsbezogene Förderquote in einer Region überschritten wird.

\title{
Hausärzte verlieren Honorar an Facharztlabor
}

_ Die bundeseinheitliche Laborquote „Q“ für das 1. Halbjahr 2016 beträgt laut KBV 91,5\%. Diese Auszahlungsquote wird damit in den beiden Abrechnungsquartalen des Halbjahres für Laboruntersuchungen der EBM-Abschnitte 32.2 und 32.3 zugrunde gelegt. Die betreffenden Laborleistungen werden in der ambulanten Versorgung also grundsätzlich nur zu diesem Prozentsatz vergütet.

Die KBV-Vorgaben sehen aber auch vor, dass für die Laborleistungen ein eigener Honorartopf gebildet wird - und zwar noch vor der Trennung der Ge- samtvergütung in einen hausärztlichen und einen fachärztlichen Teil. Es ist aber bereits klar, dass dieses Geld wegen des weiterhin sehr starken Leistungsbedarfszuwachses im Bereich Labor am Ende nicht reichen wird. Deshalb könnte man rechnerisch selbst bei voller Ausschöpfung des Topfes nur eine Quote von $84,17 \%$ auszahlen.

\section{MMW-KOMMENTAR}

Das bedeutet, das wieder einmal Geld nachgeschossen werden muss, um auf die Mindestquote von 91,5\% zu kommen. Dieses
Geld kommt gemäß den Vorgaben aus dem haus- und aus dem fachärztlichen Bereich, und zwar entsprechend der Trennungsquote. Hausärzte werden deshalb in der Regel etwa zur Hälfte an diesem Nachschussbetrag beteiligt - obgleich der größte Teil des Honorars für diese Laborleistungen Fachärzten zufließt. Streng genommen handelt es sich deshalb um einen Vorgang, der gegen den gesetzlichen Trennungsbeschluss verstößt und deshalb gesetzwidrig ist. In einigen KVen laufen deshalb bereits Widerspruchsaktionen mit dem Ziel, hier eine juristische Klärung herbeizuführen. 